\title{
Interaction-induced decoherence in non-Hermitian quantum walks of ultracold Bosons
}

\author{
K. Rapedius and H. J. Korsch \\ FB Physik, TU Kaiserslautern, D-67653 Kaiserslautern, Germany
}

(Dated: November 9, 2018)

\begin{abstract}
We study the decoherence caused by particle interaction for a conceptually simple model, a quantum walk on a bipartite one-dimensional lattice with decay from every second site. The corresponding non-interacting (linear) system has been shown to have a topological transition described by the average displacement before decay. Here we use this topological quantity to distinguish coherent quantum dynamics from incoherent classical dynamics caused by a breaking of the translational symmetry. We furthermore analyze the behavior by means of a rate equation providing a quantitative description of the incoherent nonlinear dynamics.
\end{abstract}

PACS numbers: 03.65Vf, 03.65 Yz, 03.75.Gg, 05.60.Gg, 64.70Tg

The quantum-to-classical transition often denoted as decoherence is of increasing recent interest and considerable progress has been achieved [1, 2]. Open manyparticle systems, in particular ultra-cold quantum gases and Bose-Einstein condensates, are well-suited for investigating decoherence processes in nonequilibrium quantum dynamics. Recent examples of theoretical and experimental studies of such systems include quantum walks in optical lattices 3 - 5] and the decay dynamics in open, non-hermitian systems [6-13], some of which explicitly address decoherence [11-13].

In this brief report, we analyze the mechanism of decoherence in the nonequilibrium dynamics of an interacting Bose gas by means of a topological quantity introduced in [14]. Topological properties have also been of interest in other recent investigations with cold atoms concerning, e.g., quantum Hall effects [15, 16], Berry phases [17, 18] or dissipative quantum wires [19]. We will demonstrate an interaction induced decoherence, i.e. a quantum-toclassical transition for a conceptually simple model system. In particular, we study the quantum walk of ultracold bosons in a deep bipartite 1D optical lattice with two different tunneling rates and decay from every second site described in a non-hermitian tight-binding model considered by Rudner and Levitov [14] in their analysis of a topological transition. Here we generalize and study the non-hermitian discrete Gross-Pitaevskii (or nonlinear Schrödinger) equations

$$
\begin{aligned}
\mathrm{i} \hbar \dot{a}_{m} & =\left(\epsilon_{\mathrm{a}}+g\left|a_{m}^{2}\right|\right) a_{m}-\frac{v}{2} b_{m}-\frac{v^{\prime}}{2} b_{m+1} \\
\mathrm{i} \hbar \dot{b}_{m} & =\left(\epsilon_{\mathrm{b}}-\mathrm{i} \frac{\gamma}{2}+g\left|b_{m}\right|^{2}\right) b_{m}-\frac{v}{2} a_{m}-\frac{v^{\prime}}{2} a_{m-1}
\end{aligned}
$$

where the nonlinear terms $g\left|a_{m}\right|^{2}, g\left|b_{m}\right|^{2}$ model the interaction between the particles. (Note that such an equation introduced heuristically in studies of ultra-cold bosonic a mean-field approximation from a multi-particle Bosegases or optical devices [20] can be derived rigorously as

Hubbard model with dissipation in the limit of high particle numbers in the system [12]. In a different approach, however, a mean-field limit with a renormalized interaction term appears [21, 22].) The system (11) is illustrated graphically in Fig. 1. Initially all particles occupy a single non-decaying site which corresponds to the initial conditions $a_{m}(0)=\delta_{m 0}, b_{m}(0)=0$ at time $t=0$.

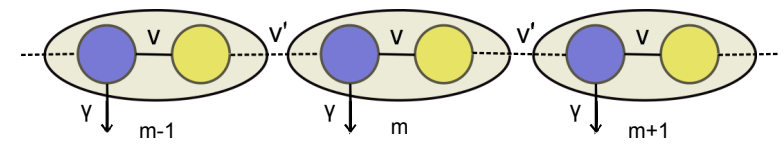

FIG. 1: (Color online) Bipartite 1D lattice with couplings $v$ and $v^{\prime}$ and decay from every second site with decay rate $\gamma$.

In the noninteracting (linear) case, (10) with $g=0$, Rudner and Levitov [14] analyzed the topological quantity

$$
\Delta m=\sum_{m} m P_{m}, \quad P_{m}=\int_{0}^{\infty} \gamma\left|b_{m}(t)\right|^{2} d t
$$

describing the average displacement of the particles before their decay. By means of a Fourier transformation to momentum space they showed that $\Delta m$ is equal to the winding number of the relative phase between components of the Bloch wave function which leads to a quantization of $\Delta m$ as a function of the ratio $v /\left(v+v^{\prime}\right)$ of the tunneling coefficients: $\Delta m=1$ for $v /\left(v+v^{\prime}\right)<1 / 2$ whereas $\Delta m=0$ for $v /\left(v+v^{\prime}\right)>1 / 2$. In [14] the quantization of $\Delta m$ was shown to be robust against intersublattice dephasing and classical noise on the energy levels $\epsilon_{a}, \epsilon_{b}$ by means of numerical simulations. This quantized behavior was compared to the continuous dependence of $\Delta m$ on $v$ and $v^{\prime}$ in the case of incoherent tunneling.

The latter can be obtained by assuming a randomly fluctuating phase between the sites so that the rates for incoherent hopping are given by perturbation theory to the lowest order as $\Gamma \propto v^{2}, \Gamma^{\prime} \propto v^{\prime 2}[23$. Initially only the site $a_{0}$ is occupied. After the very first hopping process to one of the decaying neighbour sites $b_{0}$ or $b_{1}$ corresponding to $m=0$ and $m=1$ respectively, the incoher*Electronic address: rapedius@physik.uni-kl.de, korsch@physik.uni-kl.dent dynamics between the decaying sites $b_{m}$ is symmetric 
with regard to transport to the left and right so that the contributions of all subsequent hoppings to the displacement (2) cancel out. Since the $m=0$ term does not contribute, the displacement $\Delta m$ is given by the probability of an initial hopping to site $b_{1}$ with $m=1$ which is equal to the corresponding relative hopping coefficient, i.e.

$$
\Delta m_{\text {incoherent }}=\Gamma^{\prime} /\left(\Gamma+\Gamma^{\prime}\right)=v^{\prime 2} /\left(v^{2}+v^{\prime 2}\right) .
$$

Figure 2 compares the the quantized behavior of $\Delta m$ for coherent tunneling (dashed line) with the continuous curve (3) (solid line) for incoherent tunneling.

In order to investigate the influence of a finite meanfield interaction $g \neq 0$ we integrate the system (10) numerically on a finite lattice with periodic boundaries and parameters $\epsilon_{a}=\epsilon_{b}=0$. In all numerical calculations scaled units with $\hbar=1$ are used throughout this paper. The results are also displayed in Fig. 2. Even in the linear case $g=0$ the numerical results for $\Delta m$ deviate from the perfectly quantized behavior of the idealized infinitely extended system around $v /\left(v+v^{\prime}\right) \approx 0.5$ due to effects of finite size and finite integration time. These effects were discussed in detail in 14] and are not significantly altered by the mean-field interaction. For increasing values of the interaction parameter $g$, the values of $\Delta m$ deviate more and more from the quantized behavior of the linear system, approaching the incoherent tunneling curve for higher values of $g$. Thus the mean-field interaction, unlike other kinds of disturbances mentioned above, induces decoherence in the system.

The reason for this decoherence lies in the fact that the nonlinear interaction breaks the translational symmetry within the non-decaying sublattice as will become clear in the following. To support our argument, we will demonstrate that, instead of considering interactions, the coherence observed in the linear system can also be disturbed by means of a much simpler symmetry breaking mechanism, namely by adding a constant energy shift to the initial site $a_{0}$. Figure 3 shows the numerically calculated displacement $\Delta m$ as a function of $v /\left(v+v^{\prime}\right)$ for the system (11) with $g=0$ and an additional energy shift $\eta$ of the initial site energy, i.e. we make the replacement $\epsilon_{a} \rightarrow \epsilon_{a}+\eta \delta_{m 0}$ in (11). We observe that the values of $\Delta m$ more and more approach the incoherent hopping curve for increasing values of the energy shift $\eta$. By means of a constant energy shift of a single site we are thus able to mimic the decoherence inducing effect of the mean-field interaction, thereby identifying the breaking of the translational symmetry within the non-decaying sublattice as the main cause of decoherence.

To gain further insight we analyze the time-dependence of the correlations $a_{n}^{*}(t) a_{m}(t), b_{n}^{*}(t) b_{m}(t)$ and $b_{n}^{*}(t) a_{m}(t)$ between the sites within and between the two sublattices which is most conveniently done by reexpressing our system by means of a density matrix. At first we concentrate on the simpler case of a non-interacting system with an additional shift of the initial site. For convenience, we first rewrite the system (10) with $g=0$ and an additional

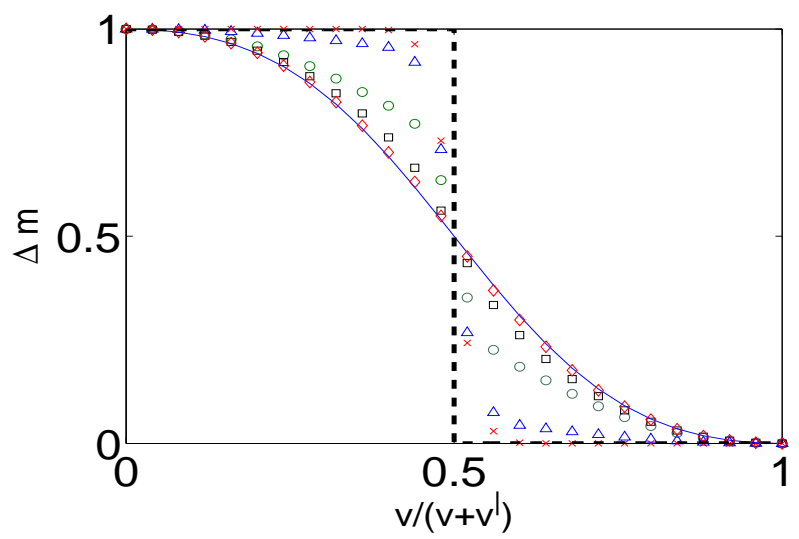

FIG. 2: (Color online) Displacement $\Delta m$ as a function of the ratio $v /\left(v+v^{\prime}\right)$ of the tunneling coefficients. '- -': linear $(g=0)$ infinitely extended system, '-' incoherent hopping according to (3). The symbols represent numerical results for a finite system with 46 lattice sites and periodic boundary conditions: ' $X$ ': $g=0$, ' $\Delta$ ': $g=0.2$, '०': $g=0.5$, ' $\square$ ': $g=1$ , $\triangleright$ ': $g=4$. The parameters in the numerical simulation are $\gamma=2, v^{\prime}=0.5$ with $0 \leq v<0.5$ for $v /\left(v+v^{\prime}\right)<0.5$ and $v=0.5$ with $0 \leq v^{\prime}<0.5$ for $v /\left(v+v^{\prime}\right)>0.5, \epsilon_{a}=0=\epsilon_{b}$.

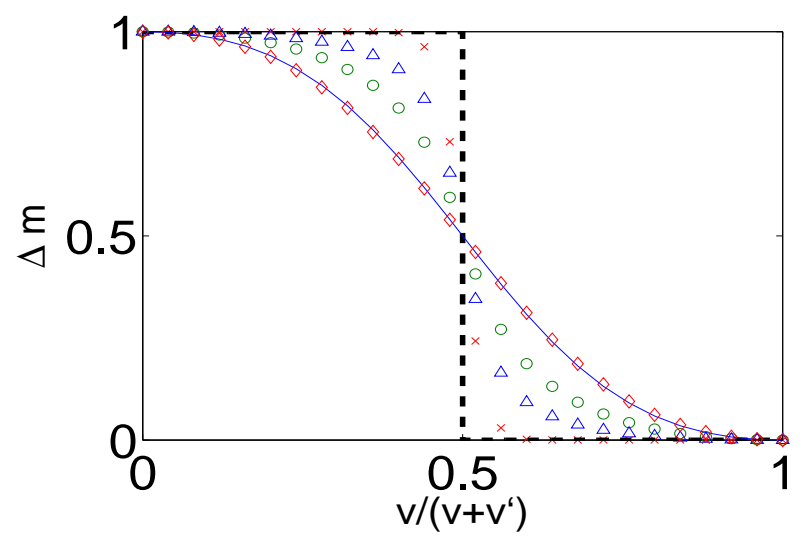

FIG. 3: (Color online) As Fig. 2 but for the linear system $(g=0)$ with an energy offset $\eta$ of the initial site. ' $X ': \eta=0$, 's': $\eta=0.05$, '॰': $\eta=0.1,{ }^{\prime} \diamond ': \eta=0.6$.

energy shift $\eta$ of the site $a_{0}$ in the compact form

$$
\mathrm{i} \hbar \dot{c}_{\alpha}=-J_{\alpha}^{\prime} c_{\alpha+1}-J_{\alpha} c_{\alpha-1}+E_{\alpha} c_{\alpha}
$$

where

$c_{\alpha}=\left\{\begin{array}{cc}a_{m=\alpha / 2}, & \alpha \text { even } \\ b_{m=(\alpha+1) / 2}, & \alpha \text { odd }\end{array}, E_{\alpha}=\left\{\begin{array}{cc}\epsilon_{\mathrm{a}}+\eta \delta_{\alpha 0}, & \alpha \text { even } \\ \epsilon_{\mathrm{b}}-\mathrm{i} \gamma / 2, & \alpha \text { odd }\end{array}\right.\right.$

and

$$
J_{\alpha}=\left\{\begin{array}{cc}
v / 2, & \alpha \text { even } \\
v^{\prime} / 2, & \alpha \text { odd }
\end{array}, \quad J_{\alpha}^{\prime}=\left\{\begin{array}{ll}
v^{\prime} / 2, & \alpha \text { even } \\
v / 2, & \alpha \text { odd }
\end{array} .\right.\right.
$$

For the dynamics of the density matrix elements $\rho_{\alpha \beta}=$ 
$c_{\alpha}^{*} c_{\beta}$ Eq. (4) then yields

$$
\begin{aligned}
\mathrm{i} \hbar \dot{\rho}_{\alpha \beta}= & \left(E_{\beta}-E_{\alpha}^{*}\right) \rho_{\alpha \beta}+J_{\alpha}^{\prime} \rho_{\alpha+1, \beta}+J_{\alpha} \rho_{\alpha-1, \beta} \\
& -J_{\beta}^{\prime} \rho_{\alpha, \beta+1}-J_{\beta} \rho_{\alpha, \beta-1} .
\end{aligned}
$$

The initial conditions at $t=0 \mathrm{read} \rho_{\alpha \beta}(0)=\delta_{\alpha \beta} \delta_{\alpha 0}$. As above we assume $\epsilon_{a}=0=\epsilon_{b}$ in the following. We consider the dynamics of the central diagonal element of the density matrix

$$
\mathrm{i} \hbar \dot{\rho}_{00}=J_{0}^{\prime}\left(\rho_{10}-\rho_{01}\right)+J_{0}\left(\rho_{-1,0}-\rho_{0,-1}\right)
$$

which depends on the nearest off-diagonal elements. As an example we have a closer look at the element

$\mathrm{i} \hbar \dot{\rho}_{01}=\left(E_{1}-E_{0}^{*}\right) \rho_{01}+J_{0}^{\prime} \rho_{11}+J_{0} \rho_{-1,1}-J_{1}^{\prime} \rho_{02}-J_{1} \rho_{00}$.

If we neglect the off-diagonal elements between two odd (i.e. decaying) sites we arrive at

$$
\mathrm{i} \hbar \dot{\rho}_{01} \approx\left(E_{1}-E_{0}^{*}\right) \rho_{01}+J_{0}^{\prime}\left(\rho_{11}-\rho_{00}\right)-J_{0} \rho_{02}
$$

where we have used $J_{1}=J_{0}^{\prime}$. The dynamics of the off-diagonal element $\rho_{02}$ must be examined more closely. Since our initial state is localized at site 0 we can neglect decaying off-diagonal elements not involving site 0 . If we additionally neglect correlations over a distance of three or more sites Eq. (77) yields i $\hbar \dot{\rho}_{02} \approx\left(E_{2}-E_{0}^{*}\right) \rho_{02}-J_{0} \rho_{01}$. Assuming a slow time-dependence of $\rho_{01}$ compared to $\hbar /\left(E_{2}-E_{0}^{*}\right)$, we obtain $\rho_{02} \approx \rho_{01}\left(1-\exp \left(-i\left(E_{2}-\right.\right.\right.$ $\left.\left.\left.E_{0}^{*}\right) t / \hbar\right)\right) J_{0} /\left(E_{2}-E_{0}^{*}\right)$. For $\left|E_{2}-E_{0}^{*}\right|=|\eta| \gg J_{0}$ the term $J_{0} \rho_{02}$ in (10) can thus be neglected. Physically this corresponds to a suppression of resonant tunneling between the sites 0 and 1 due to the large difference $\left|E_{2}-E_{0}^{*}\right|=|\eta|$ of the on-site energies. Following the reasoning in [24] we assume a slow time-dependence of $\rho_{00}$ and $\rho_{11}$ compared to the real part of $\hbar /\left(E_{1}-E_{0}^{*}\right)$, which is justified for $\left|\operatorname{Re}\left(E_{1}-E_{0}^{*}\right)\right|=|\eta| \gg J_{0}$, to obtain

$$
\rho_{01}=\frac{J_{0}^{\prime}}{\hbar} \frac{\rho_{11}-\rho_{00}}{\eta+\mathrm{i} \gamma / 2}=\rho_{10}^{*}
$$

with $E_{1}-E_{0}^{*}=-\eta-\mathrm{i} \gamma / 2$ and, analogously $\rho_{0,-1}=$ $\left(J_{0} / \hbar\right)\left(\rho_{-1,-1}-\rho_{00}\right) /(\eta+\mathrm{i} \gamma / 2)=\rho_{-1,0}^{*}$. Thus the dynamics of the central diagonal matrix element (8) becomes

$$
\dot{\rho}_{00} \approx-\left(\Gamma+\Gamma^{\prime}\right) \rho_{00}+\Gamma \rho_{-1,-1}+\Gamma^{\prime} \rho_{11}
$$

where

$\Gamma=\frac{\left(J_{0} / \hbar\right)^{2} \gamma}{\eta^{2}+\gamma^{2} / 4}=\frac{(v / \hbar)^{2} \gamma}{4 \eta^{2}+\gamma^{2}}, \Gamma^{\prime}=\frac{\left(J_{0}^{\prime} / \hbar\right)^{2} \gamma}{\eta^{2}+\gamma^{2} / 4}=\frac{\left(v^{\prime} / \hbar\right)^{2} \gamma}{4 \eta^{2}+\gamma^{2}}$.

Note the quadratic dependencies $\Gamma \propto v^{2}, \Gamma^{\prime} \propto v^{\prime 2}$ already stated before Eq. (3). For a sufficiently strong decay coefficient $\gamma$ (compared to the tunneling coefficients $v$ and $v^{\prime}$ ) one can assume that the correlations between sites further away from site 0 , which vanish at $t=0$, do not build up significantly in the course of the decay process.
As an approximation we may thus assume an incoherent dynamics as described by (12) for all sites with some local site-dependent transition rates $\Gamma_{\alpha}$ and $\Gamma_{\alpha}^{\prime}$ yielding a rate equation

$$
\begin{aligned}
\dot{\rho}_{\alpha \alpha} & \approx-\left(\Gamma+\Gamma^{\prime}\right) \rho_{\alpha \alpha} \\
& +\left\{\begin{array}{cc}
\Gamma_{\alpha} \rho_{\alpha-1, \alpha-1}+\Gamma_{\alpha}^{\prime} \rho_{\alpha+1, \alpha+1}, & \alpha \text { even } \\
-\gamma \rho_{\alpha \alpha}+\Gamma_{\alpha}^{\prime} \rho_{\alpha-1, \alpha-1}+\Gamma_{\alpha} \rho_{\alpha+1, \alpha+1}, & \alpha \text { odd } .
\end{array}\right.
\end{aligned}
$$

However, due to the decay in the system the quantities that we are interested in, namely $\Delta m$ and the occupation of the central site $\rho_{00}$ (cf. below) are not sensitive to the exact dynamics in the outer sites whose main effect in this context is the suppression of boundary effects. Thus for simplicity we make the approximation of constant transition rates $\Gamma_{\alpha}=\Gamma$ and $\Gamma_{\alpha}^{\prime}=\Gamma^{\prime}$ in the following. Within this approximation, equation (14) can be solved in closed form in Fourier space with the result (2) for $\Delta m$ (see Appendix).

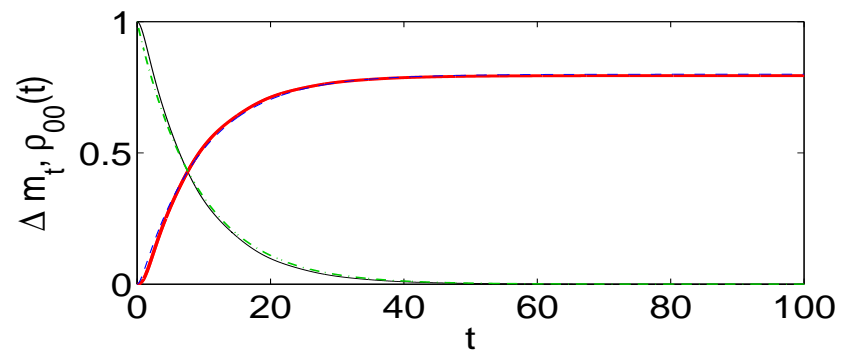

FIG. 4: (Color online) (Color online) Dynamics of the linear system $(g=0)$ with an energy offset $\eta=0.6$ of the initial site $\alpha=0$ for the parameters $v=0.25, v^{\prime}=0.5, \gamma=2$ and $\epsilon_{a}=0=\epsilon_{b}$. The numerical integration of the full system (4) ( $\rho_{00}$ :solid line, $\Delta m_{t}$ : bold solid line) is compared with the dynamics according to the rate equations (14) ( $\rho_{00}$ :dashed dotted line, $\Delta m_{t}$ : dashed line).

In order to compare the dynamics obtained from the rate equation (14) with the dynamics of the full system we define the time-dependent displacement $\Delta m_{t}$, which is obtained if the time integration in (3) is only performed up to a finite time $t$ such that $\Delta m=\lim _{t \rightarrow \infty} \Delta m_{t}$. An example of the dynamics of $\Delta m_{t}$ for $\eta=0.6$ is shown in Fig. 4 together with the corresponding decay of the central site occupation $\rho_{00}=\left|a_{0}\right|^{2}$. While $\rho_{00}$ decays exponentially, the displacement $\Delta m_{t}$ increases until it reaches its final value, given approximately by (3), for long times. For both quantities the numerically exact calculation is reasonably well approximated by the rate equation result.

Let us now return to the original, nonlinear problem with a finite interaction $g \neq 0$. The role of the energy offset $\eta$ is now played by the mean-field interaction. For a sufficiently strong decay coefficient $\gamma$, the instantaneous energy offset between the central site and its neighbors is approximately given by the local mean-field interaction term at site 0 . To obtain an approximate descrip- 

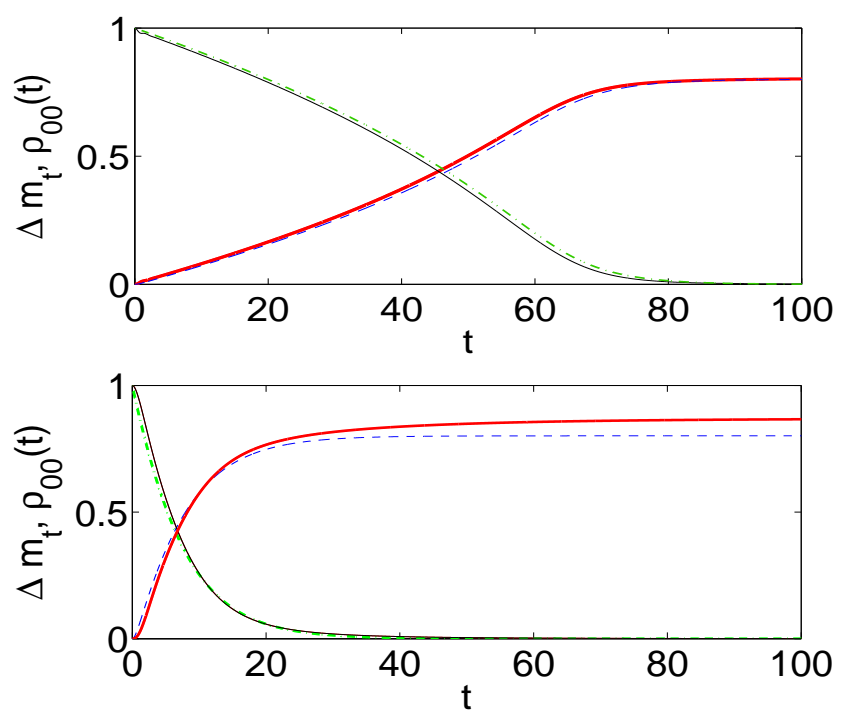

FIG. 5: (Color online) As Fig. 4 but for the nonlinear system with $g=4$ (upper panel) and $g=0.5$ (lower panel).

tion of the nonlinear system dynamics we thus use the rate equation (14) with the replacement $\eta=g \rho_{00}(t)$. Even though the hopping rates $\Gamma$ and $\Gamma^{\prime}$ are now timedependent, their ratio remains constant on this level of approximation such that the final displacement $\Delta m$ is still well approximated by (3). The upper panel of Fig. 5 demonstrates that for $g=4$, corresponding to the incoherent regime (cf. Fig. 2), the modifications of the system dynamics due to the nonlinearity are well described by the effective rate equation, both for the time-dependent displacement $\Delta m_{t}$ and the central site occupation $\rho_{00}$. The latter now shows a nonexponential decay behavior typical of open nonlinear systems [6 9]. In the long time limit, the exponential decay of the linear system is recovered as the influence of the nonlinear interaction term $g \rho_{00}(t)$ becomes negligible. For comparison the lower panel of Fig. 5 shows the dynamics for a smaller interaction $g=0.5$ corresponding to an intermediate, still partially coherent regime (cf. Fig. 2). While the decay of the central site occupation is still described reasonably well by the rate equation approach we find clear deviations for the time-dependent displacement $\Delta m_{t}$ as expected.

In summary, the interaction-induced decoherence of cold bosons spreading in a bipartite optical lattice with decay was analyzed by means of a topological quantity. Using numerical and analytical methods, the breaking of translational symmetry within the non-decaying sublattice and the resulting suppression of resonant tunneling was identified as the cause of decoherence. For the regime of strong decay and strong interaction, a rate equation providing a quantitative description of the system's incoherent nonlinear dynamics was derived. The authors hope that the present model study can contribute to a better understanding of the transition from coherent quantum dynamics to incoherent classical dynamics in mesoscopic systems.

We thank Eva-Maria Graefe and Dirk Witthaut for useful comments and suggestions.

\section{Appendix}

Changing the notation to $p_{m}^{( \pm)}=\rho_{\alpha \alpha}$ for $\alpha$ even $(+)$ or odd (-) Eq. (14) with constant decay rates $\Gamma_{\alpha}=\Gamma$ and $\Gamma_{\alpha}^{\prime}=\Gamma^{\prime}$ reads

$$
\begin{aligned}
& \dot{p}_{m}^{(+)}=-\Gamma_{0} p_{m}^{(+)}+\Gamma p_{m}^{(-)}+\Gamma^{\prime} p_{m+1}^{(-)} \\
& \dot{p}_{m}^{(-)}=-\Gamma_{0}^{\prime} p_{m}^{(-)}+\Gamma^{\prime} p_{m-1}^{(+)}+\Gamma p_{m}^{(+)}
\end{aligned}
$$

with $\Gamma_{0}=\Gamma+\Gamma^{\prime}$ and $\Gamma_{0}^{\prime}=\Gamma_{0}+\gamma$. As in [14] we switch to the momentum representation

$$
p_{m}^{( \pm)}=\frac{1}{2 \pi} \oint \mathrm{d} k \mathrm{e}^{\mathrm{i} k m} q_{k}^{( \pm)}, \quad q_{k}^{( \pm)}=\sum_{m} \mathrm{e}^{-\mathrm{i} k m} p_{m}^{( \pm)}
$$

where the integration extends over the Brillouin zone $-\pi \leq k<\pi$. The resulting equations for the momentum distributions

$$
\begin{aligned}
& \dot{q}_{k}^{(+)}=-\Gamma_{0} q_{k}^{(+)}+\Gamma_{k} q_{k}^{(-)} \\
& \dot{q}_{m}^{(-)}=-\Gamma_{0}^{\prime} q_{k}^{(-)}+\Gamma_{k}^{*} q_{k}^{(+)}
\end{aligned}
$$

with $\Gamma_{k}=\Gamma+\Gamma^{\prime} \mathrm{e}^{\mathrm{i} k}$ can be solved immediately and a solution for the initial conditions $q_{k}^{(+)}(0)=1, q_{k}^{(-)}(0)=$ 0 (the translation of $p_{m}^{(+)}(0)=\delta_{m 0}, p_{m}^{(-)}(0)=0$ ) is

$$
\begin{aligned}
q_{k}^{(+)} & =\frac{1}{\lambda_{+}-\lambda_{-}}\left(\left(\lambda_{+}+\Gamma_{0}^{\prime}\right) \mathrm{e}^{\lambda_{+} t}-\left(\lambda_{-}+\Gamma_{0}^{\prime}\right) \mathrm{e}^{\lambda_{-} t}\right) \\
q_{k}^{(-)} & =\frac{\Gamma_{k}^{*}}{\lambda_{+}-\lambda_{-}}\left(\mathrm{e}^{\lambda_{+} t}-\mathrm{e}^{\lambda_{-} t}\right)
\end{aligned}
$$

with

$$
\lambda_{ \pm}=-\left(\Gamma_{0}+\Gamma_{0}^{\prime}\right) / 2 \pm \sqrt{\gamma^{2} / 4+\left|\Gamma_{k}\right|^{2}}
$$

The momentum representation of (2) is given by

$$
\begin{aligned}
\Delta m & =\gamma \int_{0}^{\infty} d t \sum_{m} m p_{m}^{(-)}(t)=\left.\gamma \int_{0}^{\infty} d t \mathrm{i} \partial_{k} q_{k}^{(-)}(t)\right|_{k=0} \\
& =\left.\mathrm{i} \gamma \partial_{k} Q_{k}\right|_{k=0} \text { with } \quad Q_{k}=\int_{0}^{\infty} d t q_{k}^{(-)}(t) . \quad(20)
\end{aligned}
$$

Integration of the solution $q_{k}^{(-)}(t)$ in (18) yields

$$
Q_{k}=\frac{\Gamma_{k}^{*}}{\Gamma_{0} \Gamma_{0}^{\prime}-\left|\Gamma_{k}\right|^{2}} \quad \text { and } \quad \Delta m=\frac{\Gamma^{\prime}}{\Gamma+\Gamma^{\prime}} .
$$


[1] E. Joos, H. D. Zeh, C. Kiefer, D. Giulini, J. Kupsch, and I.-O. Stamatescu, Decoherence and the Appearance of the Classical World in Quantum Theory (Springer, Berlin, 2003).

[2] M. Schlosshauer, Decoherence and the Quantum-toClassical Transition (Springer, Berlin, Heidelberg, 2007).

[3] M. Karski, L. Förster, J. M. Choi, A. Steffen, W. Alt, D. Meschede, and A. Widera, Science 325, 174 (2009).

[4] D. Witthaut, Phys. Rev. A 82, 033602 (2010).

[5] C. M. Chandrashekar, Phys. Rev. A 83, 022320 (2011).

[6] P. Schlagheck and T. Paul, Phys. Rev. A 73, 023619 (2006).

[7] P. Schlagheck and S. Wimberger, Appl. Phys. B 86, 385390 (2006).

[8] K. Rapedius and H. J. Korsch, Phys. Rev. A 77, 063610 (2008).

[9] K. Rapedius and H. J. Korsch, J. Phys. B 42, 044005 (2009).

[10] K. Rapedius, C. Elsen, D. Witthaut, S. Wimberger, and H. J. Korsch, Phys. Rev. A 82, 063601 (2010).

[11] F. Gebhard, K. zu Münster, J. Ren, N. Sedlmayer, J. Sirker, and B. Ziebarth, Ann. Phys. (Berlin), doi: 10.1002/andp.201100287 (2012).

[12] F. Trimborn, D. Witthaut H. Henning, G. Kordas, T. Geisel, and S. Wimberger, Eur. Phys. J. D 63, 63
(2011)

[13] D. Witthaut, F. Trimborn, and S. Wimberger, Phys. Rev. Lett. 101, 200402 (2008).

[14] M. S. Rudner and L S. Levitov, Phys. Rev. Lett. 102, 065703 (2009).

[15] M. Hafezi, A. S. Sørensen, E. Demler, and M. D. Lukin, Phys. Rev. A 76, 023613 (2007).

[16] N. Goldman, D. F. Urban, and D. Bercioux, Phys. Rev. A 83, 063601 (2011).

[17] M. Arikawa, I. Maruyama, and Y. Hatsugai, Phys. Rev. B 82, 073105 (2010).

[18] C. B. Elsen, K. Rapedius, D. Witthaut, and H. J. Korsch, J. Phys. B 44, 225301 (2011).

[19] S. Diehl, E. Rico, M. A. Baranov, and P. Zoller, Nature Physics 7, 971 (2011).

[20] S. V. Suchkov, B. A. Malomed, S. V. Dmitriev, and Y. S. Kivshar, Phys. Rev. E 84, 046609 (2011).

[21] E. M. Graefe, H. J. Korsch, and A. E. Niederle, Phys. Rev. Lett. 101, 150408 (2008).

[22] E.-M. Graefe, H. J. Korsch, and A. E. Niederle, Phys. Rev. A 82, 013629 (2010).

[23] L. Van Hove, Physica 21, 517 (1955).

[24] A. R. Kolovsky, A. V. Ponomarev, and H. J. Korsch, Phys. Rev. A 66, 053405 (2002). 